\title{
Pion Production Data needed for Space Radiation
}

\author{
John W. Norbury * \\ NASA Langley Research Center, Hampton, Virginia, 23681, USA
}

\begin{abstract}
A recent discovery concerning the importance of hadron production in space radiation is that pions can contribute up to twenty percent of the dose from galactic cosmic ray interactions (S. Aghara, S. Blattnig, J. Norbury, R. Singleterry, Nuclear Instruments and Methods, Vol. 267, 2009, p. 1115). Although the contribution for dose equivalent will be smaller, the dose contribution could be important for fluence based radiation models. Pion production cross sections will be an essential ingredient to such models, and it is of interest to investigate the adequacy of the pion production experimental data base for energies relevant to space radiation. The pion production threshold in nucleon - nucleon reactions is at $280 \mathrm{MeV}$ and, in an interesting accident of nature, this lies near the peak of the galactic cosmic ray proton spectrum. Therefore, pion production data are needed from threshold up to energies around $50 \mathrm{GeV} /$ nucleon, where the galactic cosmic ray fluence is of decreasing importance. Total and differential cross section data for pion production in this energy range will be reviewed. The availability and accuracy of theoretical models will also be discussed. It will be shown that there are a significant lack of data in this important energy range and that theoretical models still need improvement.
\end{abstract}

\section{Nomenclature}

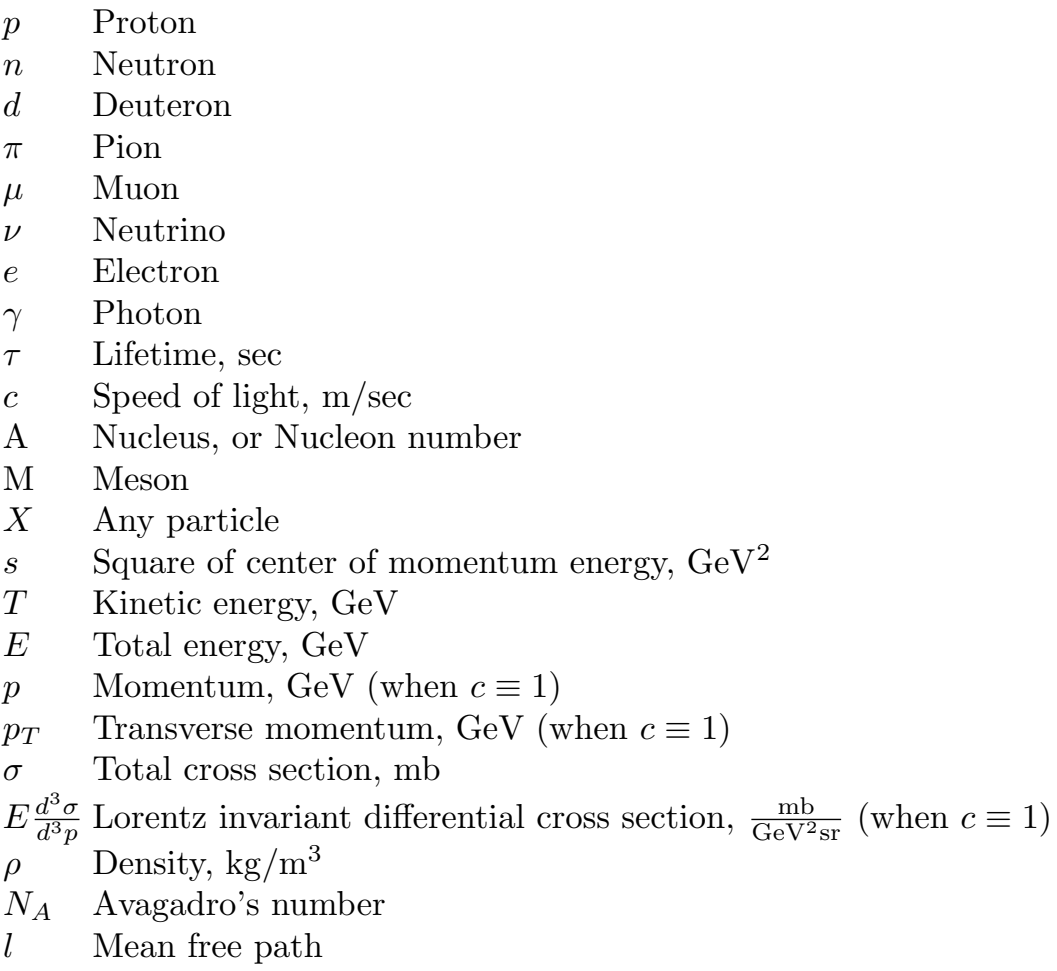

*Senior Research Scientist and Leader of the Space Radiation Group; Durability, Damage Tolerance and Reliability Branch, NASA Langley Research Center, Hampton, Virginia, 23681, USA, AIAA Senior Member. 


\section{Introduction}

The space radiation environment comprises energetic particles produced from three sources. Each source has a characteristic spectrum, which is the number of particles as a function of energy. Firstly, solar particle events (SPE) consist primarily of protons emitted from the Sun during coronal mass ejections and solar flares. These events are rare, but when they occur they can potentially inflict a lethal dose of radiation to astronauts. They are also of great concern for the stability of electronic devices. Energies can reach up to the $\mathrm{GeV}$ region. Secondly, galactic cosmic rays are protons and heavier nuclei emitted from supernovae within our Milky Way Galaxy and accelerated to the vicinity of the solar system and elsewhere. The particles have energies from $10 \mathrm{AMeV}$ out to the $\mathrm{ZeV}$ region. (Zetta $\mathrm{eV}=10^{21} \mathrm{eV}$.) The peak of the spectrum is in the $\mathrm{MeV}-\mathrm{GeV}$ region, which is most important for space radiation. For space radiation purposes, it is sufficient to consider the spectrum out to only about $50 \mathrm{GeV}$, because the flux is so much smaller beyond that energy. Thirdly, the geomagnetically trapped particles are protons and electrons confined by the magnetic field of Earth. There are two distinct regions called the inner and outer Van Allen radiation belts. Protons and electrons are found in both belts, with the most important being inner belt protons with energies up to $100 \mathrm{MeV}$ and beyond, and outer belt electrons with energies up to $100 \mathrm{keV}$ and beyond. It is important to have an accurate knowledge of the spectrum of these particles at various places in the solar system, where a particular mission will take place. The spectrum will be modified when the particles impinge on a medium. For instance, the spectrum of particles will be modified after traversal through a spacecraft wall, due to the many atomic and nuclear reactions that take place. Knowledge of the spectrum on the other side of the medium enables one to determine the dose of radiation that an astronaut receives. Several computer codes have been developed at NASA Langley Research Center that enable one to calculate the spectrum of particles on the other side of a medium, and the subsequent radiation dose. Codes at other institutions have also been developed, but they are often limited to a small number of particles and also, due to their Monte Carlo nature, take a long time to run. An important feature of the Langley codes is that they are also able to deal with the spectrum of heavy nuclei found in the galactic cosmic rays. A unique feature of the Langley codes is their short run time and ease of use, making them ideal for engineering design. The codes are deterministic and are able to run quickly allowing for almost real time simulations. Nuclei with low nucleon number provide the best protection against space radiations. This means that, for example, liquid hydrogen, water and polyethylene provide much better protection than materials such as aluminum or lead. This has major implications for human space missions and provides insight as to how to best protect astronauts and electronic devices. Transport codes are also widely used in studies of cosmic rays propagating through various media. If one knows the cosmic ray spectrum incident on top of the Earth's atmosphere, then one can deduce the cosmic ray spectrum observed on the ground by transporting through the atmosphere. Similarly, if one knows the cosmic ray spectrum incident on a spacecraft wall then one can deduce the radiation environment inside a spacecraft. ${ }^{1}$ Deterministic transport codes are concerned with solving the Boltzmann transport equation, whereas Monte Carlo codes simulate particle trajectories. HZETRN (High $\mathrm{Z}$ and Energy TRaNsport) is a computer code that is able to calculate the radiation environment inside a spacecraft, given knowledge of the external environment and the reaction cross sections. It is a deterministic space radiation transport code based upon the straight-ahead approximation. ${ }^{1}$

\section{Hadron production \& interaction}

Hadrons are produced in proton-proton, proton-nucleus and nucleus-nucleus collisions. In a high density shield, such as a spacecraft wall, the hadrons are typically absorbed before they decay. In a low density environment, such as the Earth or Mars atmosphere, the produced hadrons decay before further interactions, leading to a buildup of muons, electrons and photons, which is called an electromagnetic cascade. It has recently been found that pions can contribute up to twenty percent of the dose from galactic cosmic rays. ${ }^{2}$ (The dose equivalent has not yet been considered.) The kinetic energy threshold for pion production in proton-proton collisions is $280 \mathrm{MeV}$, and from the energies discussed previously, one can conclude that hadron production will be important for cosmic rays and possibly some energetic solar particle events, but not for geomagnetically trapped particles. Note that $p p$ cross sections are quite large. The cross section is about $50 \mathrm{mb}$ in the intermediate energy region and grows much larger as energy is increased.

It is now over seventy years since the prediction of the pion by Yukawa, and since then hundreds of other hadrons have been discovered and studied extensively. The pion is the most important hadron because 
it is the lightest, and therefore most easily produced and is responsible for the longest range part of the nucleon-nucleon interaction. One might imagine that there are no major gaps remaining in the experimental study of the pion. However, this is far from the case. The region of maximum galactic cosmic ray intensity is the $0.1-10 \mathrm{GeV}$ region, yet the energy region with the least amount of data are in the $1-10 \mathrm{GeV}$ range. This is called the intermediate energy region. Furthermore, the region where theory is least understood is $1-10 \mathrm{GeV}$ because intermediate energy is too high for non-relativistic models to work well and too low for ultrarelativistic approximations. This is made clear in the work of Eyser and Machleidt, ${ }^{3}$ who analyze spin correlation functions and show that the standard one boson exchange models, fail badly in explaining spin correlation cross section measurements. Nevertheless, they show that these models do work reasonably well when spin averages are taken into account. For space radiations studies, of course one does not need spin cross sections, but the point is that 70 years after Yukawa, there are still problems with interpreting intermediate energy measurements.

Examples of exclusive cross sections are

$$
\begin{aligned}
& p+p \rightarrow \pi^{+}+p+n, \\
& p+p \rightarrow \pi^{+}+d, \\
& p+p \rightarrow \pi^{+}+p+n+\pi^{0},
\end{aligned}
$$

where all final state particles are identified. In an inclusive cross section, these are all summed over as in

$$
p+p \quad \rightarrow \quad \pi^{+}+X
$$

where $X$ represents anything. Calculating an exclusive cross section is easier than an inclusive one, because one has to add several or many exclusive calculations to obtain the final exclusive cross section. Experimentally, it is much easier to measure only one particle, such as the $\pi^{+}$, rather than many particles.

The FLUKA and MCNPX codes both include meson production. The FLUKA code uses different interaction models depending on the energy region; below $2.5 \mathrm{GeV}$ the PEANUT (PreEquilibrium Approach to Nuclear Thermalization) model including a Generalized IntraNuclear Cascade, from 2.5 to $4 \mathrm{GeV}$ a resonance production and decay model including a Glauber Cascade, above $4 \mathrm{GeV}$ the Dual Parton Model. The DPM is based on the more general Gribov-Regge theory (GRT) formulated in terms of string interactions. The Gribov-Regge theory can be implemented in a variety of ways, which give rise to the different models such as the DPM, the Very Energetic Nuclear Scattering (VENUS) model or the Quark Gluon Strong Model (QGSM). The Los Alamos version of the quark gluon string model is called LAQGSM. The AMOR (ArtruMennessier Off-Shell Resonance) model is used in VENUS, but other possibilities are the Field-Feynman model or the Lund JETSET model. Others include multi-peripheral, parton cascade and string-parton models. One problem with many nuclear interaction models is that they use Monte Carlo procedures leading to computational inefficiency. Parametric methods can relieve the computational burden. Meson production is now included in a research version of HZETRN with pion and muon transport. The plan is to include neutral pion and eta meson production, which are the main source of the electromagnetic showers and to include charged kaons which are additional sources of muons.

Particle production in the high energy region is also well described by several types of arithmetic parameterizations, which incorporate the concept of Feynman scaling. Their advantage is that they are extremely fast to compute and are therefore suitable for transport codes, such as the deterministic code HZETRN, which run fast enough to be useful for engineering design. Therefore, studies ${ }^{4,5}$ have been made concerning the accuracy of these parameterizations for production of pions, kaons, protons and antiprotons in protonproton collisions. Figure 1 shows that these can be very accurate. Also, note that these are not mere parameterizations of data. They are based on high energy physics models incorporating important physical ideas such as scaling, which comes about due to the parton nature of hadrons. These parameterizations are also useful in applications to proton-nucleus ${ }^{4}$ and nucleus-nucleus high energy reactions. Figure 1 also shows pion production from high energy proton-Carbon collisions. Again the agreement is very good for some parameterizations. However, at lower energies, these parameterizations fail, especially in describing differential cross section measurements ${ }^{5}$ for nucleus-nucleus collisions. This is shown in Fig. 2, which also shows that a simple thermal model parameterization gives excellent results. ${ }^{5}$

In the lower energy region, FLUKA and other codes use a boson exchange model. This model does not include spin and does not include all the higher meson exchanges explicitly, but rather includes parameterizations of a simple scalar model. A model which does not include spin should really be based on 

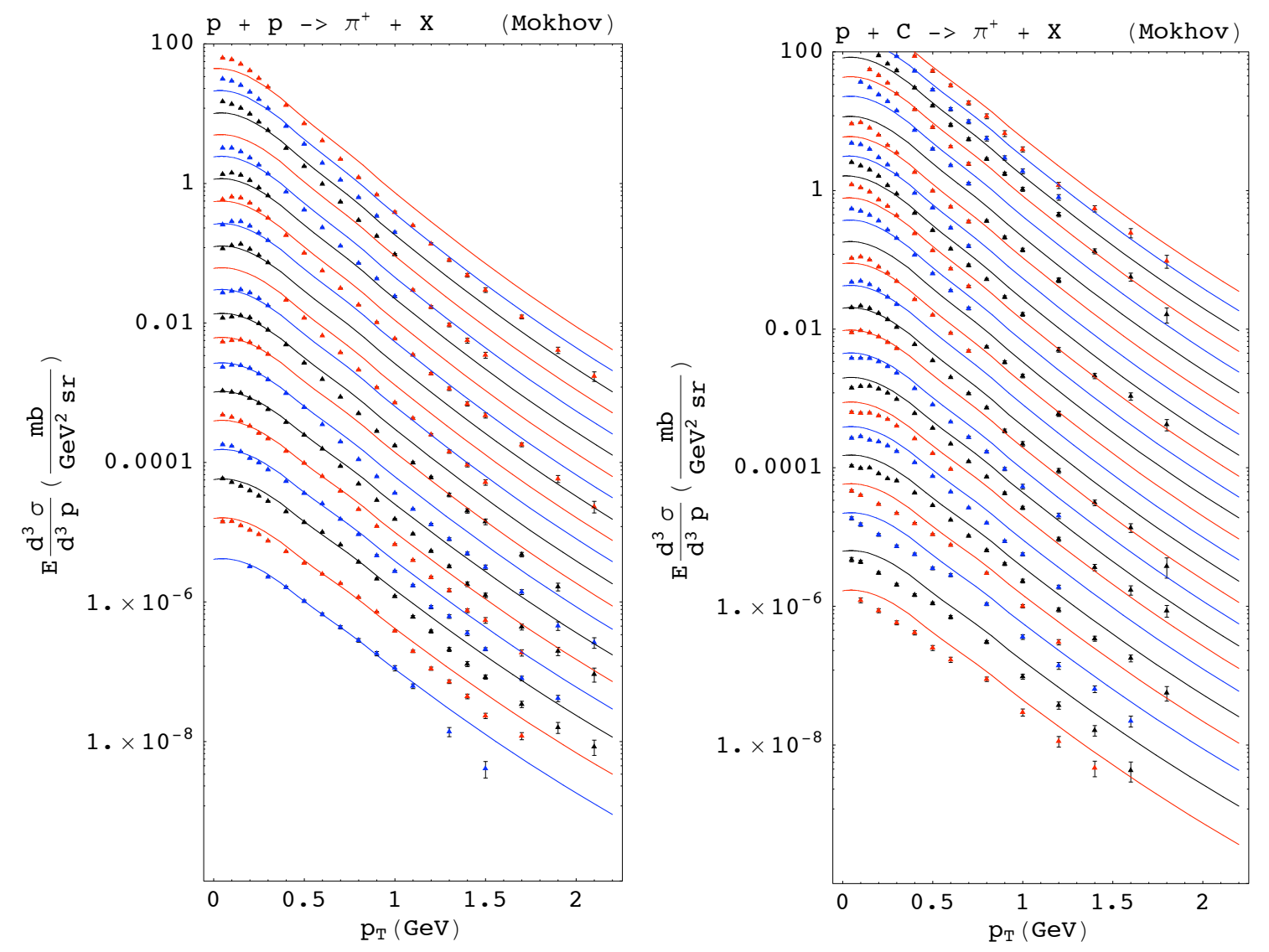

Figure 1. $\pi^{+}$production in $p p$ (left panel) and $p \mathrm{C}$ (proton-Carbon) collisions (right panel). The values of the Feynman scaling variable $x_{F}$ from top to bottom are $0.0,0.01,0.02,0.025,0.03,0.04,0.05,0.06,0.07$, $0.075,0.08,0.1,0.12,0.15,0.2,0.25,0.3,0.35,0.45,0.55$. Following reference ${ }^{6}$ data and lines are multiplied successively by 0.5 to allow for a better separation. The parameterization has been multiplied by a best fit factor of $12^{0.9}$. Data from references ${ }^{6,7}$ are plotted against the parameterization of Mokhov. ${ }^{8}$ The $p \mathrm{C}$ parameterization has been scaled by the Cronin enhancement factor as discussed in reference. ${ }^{4}$ Reproduced from reference ${ }^{4}$ with permission.

a fully consistent scalar field model and at intermediate energies one might expect such a theory to work well. Such a scalar field model is able to explain available data quite well for both $\pi$ and $\eta$ production. ${ }^{9}$ The dominant reaction mechanism for $\eta$ production is via formation and decay of the $N^{*}(1535)$ resonance through $p p \rightarrow p N^{*}(1535) \rightarrow p p \eta$. Future work will involve implementing a scalar field model ${ }^{9}$ into HZETRN, with the higher energy parameterizations being used only above intermediate energy, in a manner similar to FLUKA and GEANT, which use different models at different energies.

In the very low energy region near the pion and kaon threshold, there are interesting things to consider. ${ }^{10}$ Particle reaction transport codes, such as GEANT and FLUKA are widely used in the design of accelerator experiments and the simulation of particle detectors. While typical cross sections away from threshold reach tens of millibarn, the cross sections near threshold are about a thousand times smaller, typically in the microbarn region. The fact that theory and experiment disagreed so much in the threshold region was therefore of no real concern for particle simulation codes because the cross sections in the threshold region are so small. An interesting accident of nature is that the peak of the cosmic ray spectrum, for both protons and heavier nuclei, occurs near the pion production threshold. The Boltzmann transport equation contains a term which is the cosmic ray flux multiplied by the cross section. Therefore, when considering pion and kaon production from proton-proton reactions, small cross sections at low energy can be as important as larger cross sections at higher energy. This is also true for subthreshold kaon production in nuclear collisions, but not for subthreshold pion production. 

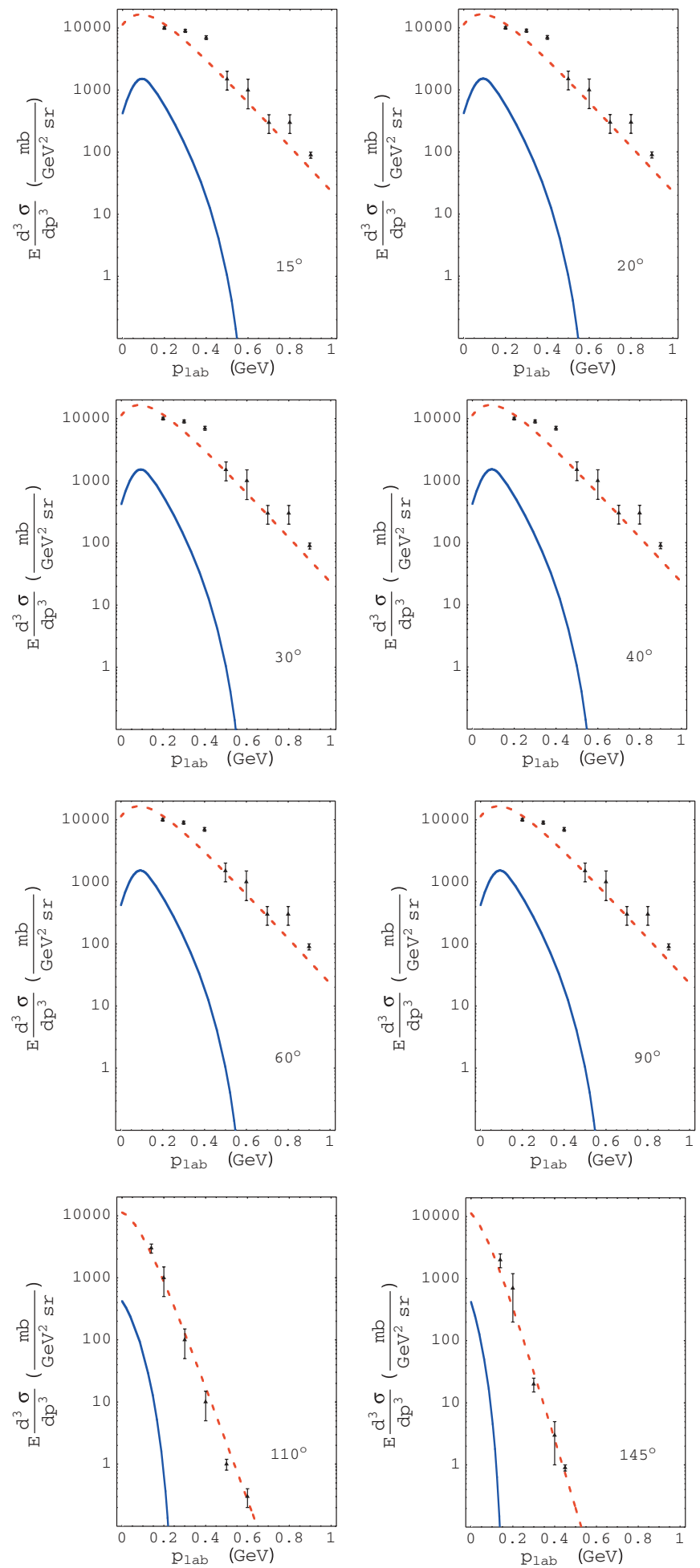

Figure 2. Inclusive $\pi^{-}$cross section for $\mathrm{Ar}+\mathrm{KCl}$ collisions. Badhwar parametrization ${ }^{11}$ (solid line) multiplied by $A_{P} A_{T}$ fails to describe data at $800 \mathrm{MeV} /$ nucleon. Lab angles are indicated. Even if the multiplication factor is changed to an arbitrary constant, the theoretical shape does not match the shape of the data. Other parametrizations are of similar poor quality. A thermal spectrum (dashed line) successfully describes data. Reproduced from reference ${ }^{5}$ with permission. 
Both the nuclear and particle reactions can be written together compactly as

$$
\begin{aligned}
A+A & \rightarrow A^{\prime}+X, \\
& \rightarrow M+X, \\
M+A & \rightarrow A^{\prime}+X \\
& \rightarrow M+X,
\end{aligned}
$$

where $A$ is any nucleus, including a proton or neutron, and $M$ is a meson such as $\pi^{+}, \pi^{-}, \pi^{0}, K^{0}, K^{+}$, or $K^{-}$. The neutral kaon contains an equal contribution from the short and long components, as in $K^{0}=$ $50 \% K_{S}^{0}+50 \% K_{L}^{0}$. Note that $A^{\prime}$ can also be $A$. The nuclear reactions (5) do not involve a meson $M$ on either the left or right side of the reactions, whereas the particle reactions (6), (7), (8) do involve $M$ on either the left or right side of the reactions. Such a compact way of writing the reactions is useful when keeping track of particles in a transport code. Note the vast number of possible reactions, because $A$ can be any nucleus, and the pions and kaons can be charged or neutral.

When pions or kaons are produced, they decay according to the following major decay modes. The charged pions undergo a weak decay ${ }^{12}$

$$
\pi^{ \pm} \rightarrow \mu^{ \pm}+\nu_{\mu} \quad(99.99 \%) \quad \tau=2.6 \times 10^{-8} \mathrm{sec}, \quad c \tau=7.8 \mathrm{~m},
$$

where $99.99 \%$ refers to the percentage of this mode of decay relative to all the possible decay modes. The lifetime $\tau$ has been listed as well as the quantity $c \tau$. Both of these quantities are in the rest frame of the decaying particle. The quantity $c \tau$ gives the approximate distance the pion will travel before decaying. This is in the rest frame of the pion. Of course in the lab frame, which is the target frame or spacecraft wall frame, the lifetime will appear longer and the distance will therefore be longer. Thus, $c \tau$ is actually a minimum distance. Given that $c \tau \approx 8 \mathrm{~m}$ for the above reaction, then the charged pion will interact and produce ionization before decaying. For this reason, one must also include the stopping powers of charged pions in radiation transport codes. The neutral pion undergoes an electromagnetic decay ${ }^{12}$

$$
\pi^{0} \rightarrow 2 \gamma \quad(99.8 \%) \quad \tau=8.4 \times 10^{-17} \mathrm{sec}, \quad c \tau=25 \mathrm{~nm} .
$$

Thus, the neutral pion can be considered not to propagate at all, but to instantaneously decay into two photons $\gamma$. These photons will immediately decay via

$$
\gamma \rightarrow e^{+}+e^{-} .
$$

The electrons will produce bremsstrahlung and ionization. The positrons will annihilate,

$$
e^{+}+e^{-} \rightarrow 2 \gamma
$$

to produce more photons which will decay, producing more electrons and positrons. Thus, a cascade of $e^{+} e^{-}$ pairs and photons will be produced from the decaying neutral pion, which can be schematically written as

$$
\pi^{0} \rightarrow \text { electromagnetic cascade } \equiv e^{+}, e^{-}, \gamma .
$$

The above considerations show why it is important to include pions and kaons in radiation transport codes. The particle flux as a function of Earth atmospheric depth steadily decreases. For typical jumbo jet altitudes (30,000 feet or $10 \mathrm{~km})$, the atmospheric depth is about $200 \mathrm{~g} / \mathrm{cm}^{2}$, whereas at altitudes appropriate for supersonic flight $(20 \mathrm{~km})$, the atmospheric depth is only about $50 \mathrm{~g} / \mathrm{cm}^{2}$. Even though neutrons are not present in the external cosmic ray spectrum, nevertheless, neutrons are dominant even at zero atmospheric depth. This is due to the neutron albedo from the atmosphere, and neutrons dominate the particle spectrum almost all the way down to the Earth surface. This, and the large relative biological effectiveness value, is why neutrons are so important for radiation received in aircraft. The reason for the monotonic fall in particle flux as a function of atmospheric depth is due to absorption from nuclear reactions. However, note that for small depths the pion and muon flux increase. Also, note that the muon flux is even larger than the proton flux at larger depths. Note that the large electron flux is due to $\pi^{0}$ decay and the associated electromagnetic cascade. (Electrons due to atomic ionization have much smaller energies.) This feature of the buildup of electromagnetic particles (electrons, photon, muons) is called an electromagnetic cascade and 
is important for radiation transport through less dense media such as Earth and Martian atmospheres. Now consider why this buildup of pions and electromagnetic particles is so important for atmospheres. It also may be important for dense media such as spacecraft shielding.

Recall that one mole is the number of atoms in a $12 \mathrm{~g}$ sample of ${ }^{12} \mathrm{C}$. This number is determined from experiment to be $N_{A}=6.02 \times 10^{23}$, which is called Avagadro's number. The interest is in nuclear reactions and so the number of nuclei is set to be approximately the number of molecules. Also, assume air to be composed entirely of Nitrogen. A $27 \mathrm{~g}$ sample of ${ }^{27} \mathrm{Al}$ or a $14 \mathrm{~g}$ sample of ${ }^{14} \mathrm{~N}$ contains $N_{A}$ molecules. Let $\bar{\rho}$ be defined as the number of nuclei per cubic centimeter,

$$
\bar{\rho} \equiv \frac{\# \text { nuclei }}{\mathrm{cm}^{3}}=\frac{\rho N_{A}}{A},
$$

where $\rho$ is the usual mass density $\left(\mathrm{g} / \mathrm{cm}^{3}\right)$ and $A$ is the atomic number. The mean free path $l$, which is the average distance between nuclear collisions, is given by ${ }^{13}$

$$
l=\frac{1}{\bar{\rho} \sigma}=\frac{A}{\rho \sigma N_{A}} .
$$

The mean free path $l$ will have units of $\mathrm{cm}$ if $\rho$ is expressed in $\mathrm{g} / \mathrm{cm}^{3}$ and $\sigma$ in $\mathrm{cm}^{2}$. Typical spacecraft shields are made from Al, tissue can be approximated as water, the Earth atmosphere can be approximated as Nitrogen, and the Mars atmosphere is mostly $\mathrm{CO}_{2}$ with a total aerial density of about $10 \mathrm{~g} / \mathrm{cm}^{2}$. For these materials,

$$
\begin{aligned}
\rho_{\mathrm{Al}} & =3 \mathrm{~g} / \mathrm{cm}^{3}, \\
\rho_{\text {water }} & =1 \mathrm{~g} / \mathrm{cm}^{3}, \\
\rho_{\text {air }} & =10^{-3} \mathrm{~g} / \mathrm{cm}^{3}, \\
\rho_{\text {Mars atmosphere }} & =10^{-5} \mathrm{~g} / \mathrm{cm}^{3}=(1 / 100) \rho_{\text {air }} .
\end{aligned}
$$

There are rough approximations made above, but only order of magnitude estimates are sought. Define

$$
\begin{aligned}
l_{1} & \equiv \frac{A}{\rho \sigma_{1} N_{A}}, \\
\sigma_{1} & \equiv 1 \mathrm{mb}=10^{-27} \mathrm{~cm}^{2},
\end{aligned}
$$

and the following results are obtained

$$
\begin{aligned}
l_{1 \mathrm{Al}} & \approx 150 \mathrm{~m}, \\
l_{1 \text { water }} & \approx 300 \mathrm{~m}, \\
l_{1 \text { air }} & \approx 200 \mathrm{~km}, \\
l_{1 \text { Mars atmosphere }} & \approx 20,000 \mathrm{~km} .
\end{aligned}
$$

At a momentum of $1 \mathrm{GeV},{ }^{12}$

$$
\begin{aligned}
\sigma_{\mathrm{pp}} & \approx 50 \mathrm{mb}, \\
\sigma_{\pi^{+} \mathrm{p}} & \approx 20 \mathrm{mb},
\end{aligned}
$$

so that

$$
\begin{aligned}
l_{\mathrm{Al}}(p p) & \approx 150 \mathrm{~m} / 50=3 \mathrm{~m}, \\
l_{\text {water }}(p p) & \approx 300 \mathrm{~m} / 50=6 \mathrm{~m}, \\
l_{\text {air }}(p p) & \approx 200 \mathrm{~km} / 50=4 \mathrm{~km}, \\
l_{\text {Mars atmosphere }}(p p) & \approx 20,000 \mathrm{~km} / 50=400 \mathrm{~km},
\end{aligned}
$$

and

$$
\begin{aligned}
l_{\mathrm{Al}}\left(\pi^{+} p\right) & \approx 150 \mathrm{~m} / 20=7.5 \mathrm{~m}, \\
l_{\text {water }}\left(\pi^{+} p\right) & \approx 300 \mathrm{~m} / 20=15 \mathrm{~m}, \\
l_{\text {air }}\left(\pi^{+} p\right) & \approx 200 \mathrm{~km} / 20=10 \mathrm{~km}, \\
l_{\text {Mars atmosphere }}\left(\pi^{+} p\right) & \approx 20,000 \mathrm{~km} / 20=1000 \mathrm{~km} .
\end{aligned}
$$


The numbers above are all rough order of magnitude estimates. Recall the $\pi^{ \pm}$decay length of $c \tau=7.8 \mathrm{~m}$. Multiplying by the relativistic $\gamma \approx 4$ factor for a $1 \mathrm{GeV}$ pion gives $\gamma c \tau \approx 30 \mathrm{~m}$. Thus, the conclusion is that in a medium such as an atmosphere, a charged pion will decay into a muon before any proton interactions occur, leading to a buildup of muons. However, in condensed matter (such as $\mathrm{Al}$ or water), this will happen less often. This is the reason for the large buildup of charged pions and muons in atmospheres. Similar arguments can be made for neutral pions $\left(\pi^{0}\right)$, which have $c \tau=25 \mathrm{~nm}$. For all types of media, these will all decay to two photons before being absorbed. Thus, for all media, $\pi^{0}$ production and decay must be considered. For a $1 \mathrm{GeV}$ pion, the photons will have energies in the $\mathrm{GeV}$ region. Consider the range of photons as a function of aerial density. $\mathrm{GeV}$ photons reach depths as high as $100 \mathrm{~g} / \mathrm{cm}^{2}$. However, photons have a relatively small biological effect compared to neutrons or heavy ions. In conclusion, neutral pion production and decay must be included for transport through shields and atmospheres. The issue of the importance of charged pions depends on the transport medium. ${ }^{2}$ Both neutral and charged pion production and decay must be included for transport through atmospheres.

\section{Total cross section for single pion production}

Apart from the neutron and proton, the pion is the most important hadron because it is the lightest meson and is therefore the most copiously produced hadron. The most fundamental observable describing its production is the total cross section for producing a single pion. Given the hundreds of other hadrons and the dozens of other experimental observables, one would assume that this fundamental observable, the total cross section for producing a single pion, is thoroughly understood. It will be shown that this is far from the case.

One of the most important conservation laws in strong interaction physics is isospin conservation, which implies the relations between cross sections as summarized in table 1. This shows that if one has experimental data for certain cross sections, then data for all particle cross sections need not be measured, because isospin conservation can be used to obtain equivalent cross sections for the remaining reactions which have not been measured. Similarly, if one does have data for these remaining cross sections, then the data can be used to test the isospin predictions. Consider the relation

$$
\sigma\left(p n \rightarrow p p \pi^{-}\right)=\sigma\left(p p \rightarrow p p \pi^{0}\right)-\frac{1}{2} \sigma\left(p p \rightarrow p n \pi^{+}\right)+\sigma\left(p n \rightarrow p n \pi^{0}\right),
$$

which is obtained from table 1 because $\sigma\left(p n \rightarrow p p \pi^{-}\right)=\sigma\left(p n \rightarrow n n \pi^{+}\right)$.

Table 1. Isospin relations between single pion production cross sections. If one assumes that data are provided for the reactions indicated, then isospin conservation provides equivalent cross sections for the remaining reactions.

\begin{tabular}{ll} 
Reaction & Equivalent cross section \\
\hline$p p \rightarrow p p \pi^{0}$ & data \\
$p p \rightarrow p n \pi^{+}$ & data \\
& \\
$p n \rightarrow p n \pi^{0}$ & data \\
$p n \rightarrow n n \pi^{+}$ & $\sigma\left(p p \rightarrow p p \pi^{0}\right)-\frac{1}{2} \sigma\left(p p \rightarrow p n \pi^{+}\right)+\sigma\left(p n \rightarrow p n \pi^{0}\right)$ \\
$p n \rightarrow p p \pi^{-}$ & $\sigma\left(p n \rightarrow n n \pi^{+}\right)$ \\
& \\
$n n \rightarrow n n \pi^{0}$ & $\sigma\left(p p \rightarrow p p \pi^{0}\right)$ \\
$n n \rightarrow n p \pi^{-}$ & $\sigma\left(p p \rightarrow p n \pi^{+}\right)$ \\
& \\
$p p \rightarrow d \pi^{+}$ & data \\
$p n \rightarrow d \pi^{0}$ & $\frac{1}{2} \sigma\left(p p \rightarrow d \pi^{+}\right)$ \\
$n n \rightarrow d \pi^{-}$ & $\sigma\left(p p \rightarrow d \pi^{+}\right)$
\end{tabular}


In order to aid the reader, table 2 gives the threshold energy for the reaction $p p \rightarrow p p \pi^{0}$, and table 3 lists the relation between kinetic energy $T$ and the root of the center of momentum totals energy $\sqrt{s}$.

Table 2. Threshold energy for $p p \rightarrow p p \pi^{0}$.

\begin{tabular}{cl} 
Threshold parameter & Value $(\mathrm{GeV})$ \\
\hline$T_{\text {lab }}$ & 0.28 \\
$E_{\text {lab }}$ & 1.2 \\
$p_{\text {lab }}$ & 0.78 \\
$\sqrt{s}$ & 2.0
\end{tabular}

Table 3. Relation between $T$ and $\sqrt{s}$.

\begin{tabular}{cc}
$\mathrm{T}(\mathrm{GeV})$ & $\sqrt{s}(\mathrm{GeV})$ \\
\hline 1 & 2.3 \\
2 & 2.7 \\
3 & 3.0 \\
4 & 3.3
\end{tabular}

Data for $\sigma\left(p p \rightarrow p p \pi^{0}\right), \sigma\left(p p \rightarrow p n \pi^{+}\right), \sigma\left(p n \rightarrow p n \pi^{0}\right), \sigma\left(p n \rightarrow p p \pi^{-}\right)$are given by Teis et al. ${ }^{14}$ and Bystricky et al. ${ }^{16}$ Cross section values, at a variety of energies, are taken from these references and are listed in table 4, which clearly shows that the experimental data are not consistent with Eq. (36). This is a very poor state of affairs for such a fundamental observable. These isospin relations are also true for differential cross sections. Transport codes, such as FLUKA and GEANT, rely on such isospin relations to calculate particle production. Data are needed to resolve this issue.

Table 4. Test of the isospin relation $\sigma\left(p n \rightarrow p p \pi^{-}\right)=\sigma\left(p p \rightarrow p p \pi^{0}\right)-\frac{1}{2} \sigma\left(p p \rightarrow p n \pi^{+}\right)+\sigma\left(p n \rightarrow p n \pi^{0}\right)$, which implies that the numbers in the last two columns should be equal.

\begin{tabular}{cccccc}
$\sqrt{s}$ & $\sigma\left(p p \rightarrow p p \pi^{0}\right)$ & $\sigma\left(p p \rightarrow p n \pi^{+}\right)$ & $\sigma\left(p n \rightarrow p n \pi^{0}\right)$ & $\sigma\left(p n \rightarrow p p \pi^{-}\right)$ & $\begin{array}{c}\sigma\left(p p \rightarrow p p \pi^{0}\right) \\
-\frac{1}{2} \sigma\left(p p \rightarrow p n \pi^{+}\right) \\
+\sigma\left(p n \rightarrow p n \pi^{0}\right)\end{array}$ \\
\hline 2.1 & 0.3 & 2 & 1 & 0.3 & 0.3 \\
2.15 & 1 & 5 & 3 & 0.5 & 1.5 \\
2.2 & 3 & 12 & 7 & 1.5 & 4 \\
2.3 & 3.5 & 17 & 14 & 2.2 & 1.7 \\
2.4 & 3.7 & 18 & 7 & 2.5 & 1 \\
2.5 & 3.5 & 17 & 7 & 2.6 &
\end{tabular}

\section{Summary of pion data}

Consider the status of experimental data for pion production. Even though there are hundreds of other particles to consider, the poor situation for pions illustrates the problem for all particles, especially as pions are better measured and understood compared to other particles. Table 5 summarizes the available data for pion production in nucleon - nucleon collisions. This will now be considered. In the discussion below, all energies refer to kinetic energy (i.e. $T$, not $\sqrt{s}$ ) unless otherwise stated. 


\section{A. Exclusive single pion production}

\section{Total cross section}

A lot of the data are summarized in the references. ${ }^{14-17}$ While there are quite a few measurements available, the following problems are noted. Many data do not go beyond a few GeV in kinetic energy. All exclusive channels have not been measured. Of the exclusive channels that are measured, some of the measurements appear inconsistent with isospin conservation, which is used in codes such as FLUKA to calculate some cross sections. Single pion production data, ${ }^{14}$ which have been shown above to be inconsistent with isospin conservation. Also, note that much of the data are for low values of kinetic energy. There are also difficulties in predicting data near threshold. ${ }^{18}$

\section{Differential cross section}

The isospin relations in table 1 also hold for differential cross sections, which need to be checked to ensure that they do not violate isospin constraints. There are very few data and most are below $1 \mathrm{GeV}$. Again, there are difficulties near threshold. ${ }^{18}$ Although, not of interest for space radiation, Eyser and Machleidt ${ }^{3}$ have shown that meson exchange models are unable to predict spin correlation functions in the GeV region.

\section{B. Exclusive multiple pion production}

A lot of data are summarized in the references. ${ }^{16,17,19}$ The experimental situation for total cross section is poor with few measurements available. There do not appear to be any data available for differential cross sections.

\section{Inclusive pion production}

Inclusive measurements are much easier than exclusive measurements, and one would think that the data situation is much better. However, most of the data are at very high energy. In the intermediate energy region $(1-10 \mathrm{GeV})$, there are very few measurements of either total or differential cross sections. This is not a good situation for space radiation, since it is the inclusive cross sections in the intermediate energy region that are most often required. One is therefore forced to use high energy parameterizations and hope that they work at intermediate energy. Note that these problems in the intermediate energy region are not of much concern for many transport codes, such as GEANT and FLUKA, when used for accelerator and detector design for particle physics experiments, because the intermediate energy region is small, and there are many other energy regions which are just as important. However, for space radiation purposes, where the cosmic ray spectrum peaks at intermediate energy, then these problems are very important. The discussion has focused on pion production and the situation is worse for the other hadrons.

Table 5. Summary of pion production data from nucleon - nucleon collisions. Single, multiple and inclusive refers to single pion production, multiple pion production and inclusive pion production.

\begin{tabular}{lll} 
& Total cross section & Differential cross section \\
\hline Single & Isospin inconsistency & Isospin needs verification \\
& $\begin{array}{l}\text { No data at several GeV } \\
\text { Threshold difficulties }\end{array}$ & $\begin{array}{l}\text { Few data mostly below 1 GeV } \\
\text { Threshold difficulties } \\
\text { Spin correlation problems }\end{array}$ \\
Multiple & Similar to Single & Few data \\
& & \\
Inclusive & Few data at 1-10 GeV & Few data at 1-10 GeV \\
& Most data at very high energy & Most data at very high energy
\end{tabular}




\section{Conclusions}

The present work has considered the availability of pion production data as well as physically motivated, parametric fits to such data. It is found that high energy parametrizations do not fit the intermediate energy data well, and instead thermal models are more suitable. There are serious gaps in the pion data, with the most important being that the data are inconsistent with isospin conservation. More precise data are needed as well as inclusive data in the intermediate energy region. Dose contributions from other hadrons also need to be evaluated.

\section{References}

${ }^{1}$ Wilson, J.W., Townsend, L.W., Schimmerling, W.S., Khandelwal, G.S., Khan, F., Nealy, J.E., Cucinotta, F.A., Simonsen, L.C., Shinn, J.L., and Norbury, J.W., "Transport methods and interactions for space radiations," NASA RP-1257, 1991.

${ }^{2}$ Aghara, S.K., Blattnig, S.R., Norbury, J.W., and Singleterry, R.C., "Monte Carlo analysis of pion contribution to absorbed dose from galactic cosmic rays," Nuclear Instruments and Methods in PHysics Research B, Vol. 267, 2009, pp.1115-1124.

${ }^{3}$ Eyser, K.O., Machleidt, R., and Scobel, W., "Modelling nucleon-nucleon scattering above 1 GeV," European Physical Journal A, Vol. 22, 2004, pp. 105-117.

${ }^{4}$ Norbury, J.W., and L. W. Townsend, L.W., "Parameterizations of inclusive cross sections for pion production in protonproton collisions. II. Comparison to new data," Physical Review D, Vol. 75, 2007, p. 034001.

${ }^{5}$ Norbury, J.W., "Pion cross section parametrizations for intermediate energy, nucleus-nucleus collisions," Physical Review C, Vol. 79, 2009, p. 037901.

${ }^{6}$ Alt, C., et al., "Inclusive production of charged pions in $\mathrm{p}+\mathrm{p}$ collisions at $158 \mathrm{GeV} / \mathrm{c}$ beam momentum," European Physical Journal C, Vol. 45, 2006, pp. 343-381.

${ }^{7}$ Alt, C., et al., "Inclusive production of charged pions in $\mathrm{p}+\mathrm{C}$ collisions at $158 \mathrm{GeV} / \mathrm{c}$ beam momentum," European Physical Journal C, Vol. 49, 2007, pp. 897-917.

${ }^{8}$ Mokhov, N.V., and Striganov, S.I., Workshop on the front end of muon collider, CP-435, 1998, pp. 453-459.

${ }^{9}$ Norbury, J.W., Dick, F., Norman, R.B., and Maung K.M., "Cross sections from scalar field theory," Canadian Journal of Physics, Vol. nnn, 2010, pp. nnn-nnn.

${ }^{10}$ Norbury, J.W., Townsend, L.W., and Norman, R.B., "Threshold meson production and cosmic ray transport," Journal of Physics G, Vol. 34, 2007, pp. 115-121.

${ }^{11}$ Badhwar, G.D., Stephens, S.A., and Golden, R.L., "Analytic representation of the proton-proton and proton-nucleus cross sections and its application to the sea level spectrum and charge ratio of muons." Physical Review D, Vol. 15, 1977, pp. 820-830.

${ }^{12}$ Amsler, C., et al., "Review of particle physics," Physics Letters B, Vol. 667, 2008, pp. 1-1340.

${ }^{13}$ Cottingham, W.N., and Greenwood, D.A., "An introduction to nuclear physics," $2^{\text {nd }}$ ed., Cambridge University Press, Cambridge, United Kingdom, 2001.

${ }^{14}$ Teis, S., Cassing, W., Effenberger, M., Hombach, A., Mosel, U., and Wolf, G., "Pion production in heavy ion collisions at SIS energies," Zeitschrift für Physik A, Vol. 356, 1997, pp. 421-435.

${ }^{15}$ Cassing, W., Metag, V., Mosel, U., and Niita, K., "Production of energetic particles in heavy ion collisions," Physics Reports, Vol. 188, 1990, pp. 363-449.

${ }^{16}$ Bystricky, J., La France, P., Lehar, F., Perrot, F., Siemiarczuk, T., and Winternitz, P., "Energy dependence of nucleonnucleon inelastic total cross sections," Journal de Physique, Vol. 48, 1987, pp. 1901-1924.

${ }^{17}$ Garcilazo, H., and Mizutani, T., " $\pi$ NN Systems," World Scientific, Singapore, 1990.

${ }^{18}$ Hanhart, C., "Meson production in nucleon-nucleon collisions close to the threshold," Physics Reports, Vo. 397, 2004, pp. 155-256.

${ }^{19}$ Nara, Y., Otuka, N., Ohnishi, A., Niita, K., and Chiba, S., "Relativistic nuclear collisions at 10A GeV energies from p + Be to $\mathrm{Au}+\mathrm{Au}$ with the hadronic cascade model," Physical Review C, Vol. 61, 1999, p. 024901. 